\title{
A model for cellular mechanotransduction and contractility at
}

\section{finite strain}

\author{
N. Bouklas*1,3 | M.S. Sakar ${ }^{1,2} \mid$ W.A. Curtin ${ }^{1}$
}

\footnotetext{
${ }^{1}$ Institute of Mechanical Engineering, Ecole Polytechnique Fédérale de Lausanne,

Lausanne, Switzerland

${ }^{2}$ Institute of Bioengineering, Ecole

Polytechnique Fédérale de Lausanne,

Lausanne, Switzerland

${ }^{3}$ Mechanical and Aerospace Engineering,

Cornell University, Ithaca, New York, USA
}

\section{Correspondence}

*Nikolaos Bouklas, Email:

nb589@cornell.edu

\section{Present Address}

Nikolaos Bouklas, Cornell University, 411

Upson Hall, Ithaca, New York 14853.

\begin{abstract}
Summary
In this work we introduce a theoretical and computational modeling framework for the contractile response of single cells triggered by external mechanical stimuli. The structural response due to the formation and dissociation of stress fibers is modeled following contractile phases with an orientation and intensity that evolves with time and strain. The passive and active structural components are postulated to act in parallel, and the re-orientation process drives the anisotropic phase of stress fiber orientation to align with the direction of the maximum principal stretch. A reduced form of the Hai-Murphy model is used to follow kinetics of myosin states considering the combined effect of "latch"- and "cross"-bridge states. The introduction of distinct isotropic and anisotropic activation allows modeling of the contractile intensity of each phase. Tractions on the cell surface initiate bio-chemical signaling through the RhoA pathway, which in turn controls both myosin contraction and F-actin polymerization. A signaling model is introduced to effectively connect intracellular events with the tractions on the cell surface. The overall model is defined by a free energy density function that couples the deformation and the activation, and associated equilibrium and kinetic models for evolution. Features of the model are highlighted via implementation in a finite element model and application to benchmark problems. The model captures the dynamic contractile responses of cells and stress fiber re-alignment under complex load histories. For example, physiologically relevant scenario such as relaxation of cells to their initial state upon removal of applied loads can be simulated.
\end{abstract}

\section{KEYWORDS:}

cell contractility, stress fibers, mechanotransduction, intracellular signaling 


\section{1 | INTRODUCTION}

Complex and often coupled chemical, mechanical and electrical phenomena regulate biological function including cellular contractility. Cellular contractility develops through sensing of microenvironment and processing of the collected information by intracellular signaling pathways 1 . Thus, in order to discern the role of contractility in development, homeostasis and disease progression, it is crucial to examine the underlying sensing-computation-actuation cycle ${ }^{[2]}$ [3] Mechanotransduction regulates the biophysical responses of several types of cells that exhibit contractile behavior, including fibroblasts, cardiomyocytes, epithelial cells, endothelial cells and smooth-muscle cells ${ }^{4}$. In this work we develop a theoretical and numerical model of a single cell as an active material that captures the mechano-chemical coupling that regulates contractility. We focus on the scenario where the response is induced through transmission of mechanical forces and consequent structural changes in the focal adhesion complex.

There has been a long history of experimental studies on the contractile behavior of living cells under static loading conditions ${ }^{[5]}$ [6, which more recently extended to cyclic loading conditions ${ }^{[7}[8]$. In the majority of these experiments, cells were plated on a two dimensional (2D) substrate to study the formation and structure of cytoskeleton as a function of loading conditions and substrate material properties. To elucidate the effect of stiffness, elastic substrates with tunable stiffness were utilized 9 . These studies confirmed the tendency of the cells to migrate towards areas of higher stiffness ${ }^{[10]}$. Recent advances in imaging and biomaterials research brought bioengineering platforms that can be employed for measuring cellular responses in a three dimensional (3D) context ${ }^{11}$. Highly coordinated multi-field and multi-scale processes are evident in these 3D tissue constructs $[12]$.

A number of coupled bio-chemical and mechanical signals contribute to the coordination of the mechanotransduction process ${ }^{[13]}$. Focal adhesions form between ligands on the extra-cellular matrix (ECM) and the integrin receptors on the cell surface. These connections allow for load transfer from the ECM to the cytoskeleton, and vise-versa when contractile forces are generated by the cell. The conformational changes of the focal adhesions are responsible for sensing of mechanical forces [14] [15]. The next step of the process is intracellular signaling, which is facilitated through multiple pathways depending on the type of cell and stimulus. The major pathway controlling stress fiber formation and contractility in non-muscle cells involves Rho signaling [16] [17]. Transmembrane adhesion receptors such as integrins sense biomechanical changes at the cell periphery, which in turn regulates the formation of focal adhesion complex that includes several proteins such as vinculin, paxilin, focal adhesion kinase and talin ${ }^{[18}$. Focal adhesion signaling controls the release of GEFs and GAPs, and these proteins regulate the activity of Rho GTPases. RhoA controls polymerization of F-actin and the myosin contraction/attachment cycle (even at fixed $\mathrm{Ca}^{2+}$ concentration). The actomyosin bundling events lead to the formation of stress fibers and development of contractile forces 19 . 
These forces in turn, are carried through the adhesion complexes back to the ECM. Increase in contractility also initiates further maturation of focal adhesions, thus closing the cycle of mechanotransduction in the cell-ECM system [20]. Finally, dynamic changes in the distribution and strength of mechanical loading play an important role in the evolution of stress fiber alignment, which in turn determines the transmission of contractile forces ${ }^{[21]}$. The main features that a model for mechanotransduction and contractility should capture, are a) the response to external mechanical stimuli by activating stress fiber formation. b) adaptive stress fiber alignment depending on the loading state and c) stress fiber dissociation upon removal of external loads.

Theoretical and computational modeling of the complete mechano-transduction cycle can be separated into three main parts: 1) the active material that includes cross-bridge dynamics and stress fiber realignment, 2) intracellular signaling and 3) the focal adhesions. Previous work on active material models can be categorized in purely phenomenological models [22] [23] [24] [25] [26] and thermodynamically motivated models 227,28$]$. In the majority of previous reports, signaling was induced by chemical or electrical stimuli. This approach has been established for the modeling of muscle cells, where the triggering signal leads to an increase of cytosolic $\mathrm{Ca}^{2+}$ concentration that drives contraction [29] [30] 31] 32]. Models of muscle cells are often not concerned with the evolution of stress fiber alignment because muscle cells have a relatively fixed intracellular sarcomeric structure and the primary focus has been on cross-bridge dynamics. Models that follow Deshpande et al. [23] including [24] [26] assume Hill's law for muscle contraction for the individual stress fibers ${ }^{33}$. The activation essentially has a permanent "latch"-bridge state, and thus show residual deformations upon equilibration once the signal completely fades. More recently, Shenoy et al. ${ }^{[27]}$ have presented a framework equipped with mechanosensory input embedded in the active material model. However, the constitutive model was developed starting from an incremental form for the energy and certain assumptions were taken for the initial state, which together led to some thermodynamic inconsistencies. Furthermore, predicted steady-state response is difficult to rationalize in physical terms. Modeling of focal adhesion has been a topic of study including recent thermodynamically motivated work by Deshpande et al. [34] [28. Following the active material model and focal adhesion models by Deshpande et al. [23] [34], Pathak et al. ${ }^{35]}$ derived a cooperative signaling model by integrating all the three parts mentioned above. This feat was achieved by connecting the configurational state and rate of formation of focal adhesions with the signaling mechanism. However, this work is restricted to one-dimensional (1D) problems and small deformations that connects the configurational state and the rate of formation of focal adhesions with the signaling mechanism.

In this work, we establish the basis for a thermodynamically consistent model that allows for 1) sensing and transduction of mechanical input, and 2) stress fiber formation and dissociation that is controlled by the chemical kinetics of different states of myosin and by the state of deformation (principal stretches). Measurements of the independent contributions of focal adhesion complexes during mechanotransduction process is a challenging task because the adhesion of cells to the ECM changes dynamically along with the level of contractility and external loading. In a modeling and computational framework, a first step 
towards incorporating the function of focal adhesions is the establishment of the contribution of tractions. Tractions must eventually influence the active contractile response through the associated signaling pathway. This way the model can recapitulate the complete cycle and allow the study of challenging fundamental problems within a coupled multi-physical framework. The work is organized as follows. In Section 2 the theoretical framework for the model is presented. In Section 3 a specific form of free energy is introduced and the resulting constitutive laws are presented. In Section 4 some benchmark problems are numerically studied and discussed. In Section 5, a concluding remark is presented, which is supported by a discussion on future directions.

\section{2 | THEORETICAL FRAMEWORK FOR CELL CONTRACTILITY}

In this section, the theoretical framework for a model that can capture the fundamental features of loading-dependent contractility of a single cell with evolving stress fiber distribution and contraction intensity is presented. Following the chemical kinetics of the states of myosin, we connect the rate of creation of "active" myosin to the tractions acting on the cell surface which are related to the activity of RhoA. We assume a homogeneous distribution of states throughout the cytoplasm. We also introduce an activation that characterizes the contractility of the stress fibers, and connect both the myosin cycle and the F-actin polymerization cycle as they are both regulated by the activity of RhoA. Regarding the stress fiber orientations, we postulate the split of the stress fiber distribution to an isotropic and an anisotropic phase, where the later captures the average orientation of the aligned stress fibers beyond the isotropic state. Only one explicit family of fibers is tracked by the the anisotropic phase. Each of the two stress fiber orientation states are related to an activation component that is a function of the local deformation state and the normalized concentration of "active" myosin. An evolution law for the average direction of the stress fibers of the anisotropic state is introduced. These components of the model are necessary to capture loading dependent formation/dissociation and alignment/re-orientation of stress fibers. The details of the derivation of these components will be discussed in the following sub-sections.

\section{1 | Chemical kinetics}

In fibroblasts, cardiomyocytes, endothelial and epithelial cells, contractility occurs due to consecutive cycles of a) phosphorylation of myosin, followed by b) attachment to actin fibers, c) a "power-stroke" action which is followed by de-phosphorylation of myosin and d) unbinding from actin fibers. The phosphorylation of myosin is driven by signaling through the level of RhoROCK activity. In this work we follow the concentration of myosin states and connect them to the signaling process of RhoA. RhoA signaling also controls F-actin polymerization. In the seminal work of Hai and Murphy ${ }^{[36}$, a chemical kinetics model of myosin states was utilized, which tracked the concentration of four states of myosin including a "latch"-bridge state. Latchbridge state explains the prolonged exertion of contractile forces at low energy consumption in smooth muscle cells. In this 
work, we present a reduced version of Hai and Murphy model, following two states of myosin (active and inactive) without differentiating between the "latch"-bridge and the "cross"-bridge state. Our general framework allows for the use of both chemical kinetics models but we focus on the later.

The Hai and Murphy model distinguishes between active and inactive configurations (bound and unbound), and between phosphorylated and de-phosphorylated states of myosin. The "cross"-bridge refers to the bound/phosphorylated state, and the "latch"-state to the bound/de-phosphorylated state. The notation used here for the normalized concentration of each state over the total myosin concentration point-wise, is $c_{i m}$ for unbound/de-phosphorylated myosin (inactive), $c_{i m p}$ for unbound/phosphorylated myosin (inactive), $c_{a m}$ for bound/de-phosphorylated myosin (active) and $c_{a m p}$ for bound/phosphorylated myosin (active). The reaction model is in the form of a system of linear coupled ordinary differential equations, and can be considered as a governing law for myosin conservation without considering any sources, sinks and transfer of myosin. The constants $\kappa_{1}$ where $i=1, . ., 7$ refer to the rates at which each chemical state of myosin transitions to another state, and the relations are obvious in 1 e.g. $\kappa_{2}$ is the rate at which $c_{i m p}$ transitions to $c_{i m}$.

$$
\frac{d}{d t}\left[\begin{array}{c}
c_{i m} \\
c_{i m p} \\
c_{a m p} \\
c_{a m}
\end{array}\right]=\left[\begin{array}{cccc}
-\kappa_{1} & \kappa_{2} & 0 & \kappa_{7} \\
\kappa_{1} & -\kappa_{2}-\kappa_{3} & \kappa_{4} & 0 \\
0 & \kappa_{3} & -\kappa_{4}-\kappa_{5} & \kappa_{6} \\
0 & 0 & \kappa_{5} & -\kappa_{6}-\kappa_{7}
\end{array}\right]\left[\begin{array}{c}
c_{i m} \\
c_{i m p} \\
c_{a m p} \\
c_{a m}
\end{array}\right]
$$

As the concentrations $c_{(. .)}$are normalized over the total concentration of myosin, the sum of normalized concentrations has to equal unity

$$
c_{i m}+c_{i m p}+c_{a m p}+c_{a m}=1 \text { first }
$$

and all individual concentrations have to be positive and less than unity

$$
0<c_{i m}, c_{i m p}, c_{a m p}, c_{a m}<1
$$

The conservation of total myosin can also be expressed in terms of the rates of change of the concentration which follows directly from Eq. 2

$$
\dot{c}_{i m}+\dot{c}_{i m p}+\dot{c}_{a m p}+\dot{c}_{a m}=0
$$

Towards building an accurate active material model for the contractile behavior of single cells, the chemical kinetics of active and inactive myosin states have to be accurately captured. In smooth-muscle cells where structures are permanent and there is no formation/dissociation of stress fibers, a "latch"-bridge state ${ }^{[29}$ of acto-myosin is what allows for the low energy prolonged exertion of contractile forces and is essential to the understanding of the contractile response of muscles. For the cell types that we are interested in this work, stress fibers form, dissociate and re-form in new directions upon change of loading 
conditions. Even though "latch"-bridges are present, their effect becomes harder to distinguish in conjunction with stress fiber re-alignment. Thus, in this work we introduce a reduced chemical kinetics model, directly derived from the Hai and Murphy model of Eq. 1 that tracks myosin concentrations but distinguishes only between active and inactive states. The notation $\tilde{c}_{a m}$ and $\tilde{c}_{i m}$ is used accordingly for the active and inactive myosin concentrations. The assumption is that we track the combined effect of "cross"-bridges and "latch"-bridges. The system of ODEs now becomes

$$
\frac{d}{d t}\left[\begin{array}{c}
\tilde{c}_{i m} \\
\tilde{c}_{a m}
\end{array}\right]=\left[\begin{array}{cc}
-k_{1} & k_{2} \\
k_{1} & -k_{2}
\end{array}\right]\left[\begin{array}{l}
\tilde{c}_{i m} \\
\tilde{c}_{a m}
\end{array}\right]
$$

where $k_{1}$ and $k_{2}$ can be directly derived by $\kappa_{i}(i=1,4)$. Using the conservation constraint

$$
\tilde{c}_{i m}+\tilde{c}_{a m}=1
$$

leads to a single ODE which can be easily formulated as

$$
\dot{\tilde{c}}_{a m}=k_{1}\left(1-\tilde{c}_{a m}\right)-k_{2} \tilde{c}_{a m}
$$

A constraint on the magnitude of the individual concentration should always be considered during the numerical solution process

$$
0<\tilde{c}_{i m}, \tilde{c}_{a m}<1
$$

When no external forces are acting on the cell, $k_{1}=k_{0}$ is the base rate of at which inactive myosin is converting to active.

\section{2 | Matrix and fiber kinematics}

Before connecting the myosin concentrations and kinetics to the development of stress fibers and contractile response, we need to first define the model of kinematics/deformation for the cell. The complete mechanical response of a contractile cell depends on the coupling of the active stress fibers and the network of microtubules of the cytoskeleton which provides the passive elastic response. In a homogenized three-dimensional cell, points $X \in \Omega_{0}$ from the reference configuration $\Omega_{0}$ can be mapped to points $\chi=\chi(X, t) \in \Omega$ on the current configuration $\Omega$ through a time-dependent map $\chi: \Omega_{0} \times \mathbb{R} \rightarrow \Omega$ and the deformation gradient can be defined as $\boldsymbol{F}=\nabla_{X}(\chi)$. The reference configuration $\Omega_{0}$ is the three-dimensional domain that corresponds to an unloaded fully passive state, with no external loads and no stress fibers. It is crucial to differentiate the reference configuration $\Omega_{0}$ from the initial configuration $\Omega^{\text {init }}$, which corresponds to a free-homeostatic state, as shown schematically in Fig. 1 The initial configuration is homogeneously deformed relative to the reference configuration, due to the action of the active isotropic distribution of stress-fibers even in the absence of any externally applied load. The initial state is thus the observed state of the isolated cell. The initial deformation is characterized by a deformation gradient defined as $\boldsymbol{F}^{\text {init }}=\lambda^{\text {init }} \boldsymbol{I}$, where $\boldsymbol{I}$ is the second order identity tensor. All quantities with a superscript ()$^{\text {init }}$ refer to the initial state. 


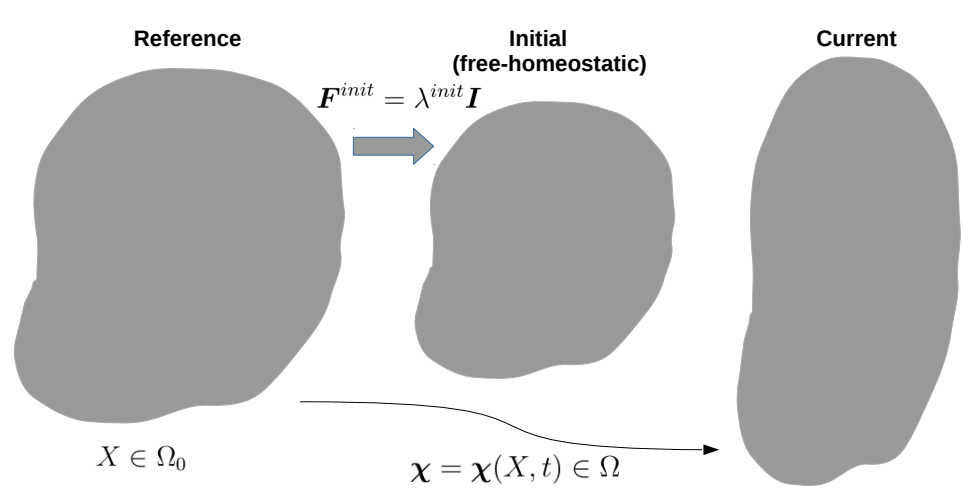

FIGURE 1 Schematic of the reference state, the initial (free-homeostatic) and the current state

In our formulation, consistent with other recent models for similar cell types [23], [27], the cytoskeleton and the active contractile components are considered to act in parallel. The deformation gradient $\boldsymbol{F}$ is thus equal to both the active deformation gradient $\boldsymbol{F}_{a}$ due to the contraction of acto-myosin motors and the passive deformation gradient $\boldsymbol{F}_{p}$ due to the elasticity of the cytoskeleton, $\boldsymbol{F}=\boldsymbol{F}_{a}=\boldsymbol{F}_{p}$. This formulation is used since the stress fibers in the type of cells considered here are not permanent constructions. This model differs from those for smooth-muscle contraction by Stalhand et al. $\sqrt{29 \sqrt{31}}$ and Murtada et al. wherein a multiplicative decomposition of the passive and active deformation gradients is used to account for the relative sliding of the stress-fiber filaments. Such an approximation is not trivial to adopt for concurrent stress fiber formation/dissociation/reorientation, and also not directly applicable since the structure of the stress-fibers evolves and is not ordered as in smooth-muscle cells.

We then model the distribution of stress-fibers as a combination of an isotropic phase and an anisotropic phase. The anisotropic phase corresponds to the average orientation of the active aligned stress-fibers, those not part of the isotropic phase, and we consider only one single anisotropic fiber direction. The two different types of active mechanisms are visualized in fig. 2 . The orientation of the anisotropic stress-fibers evolves as stress-fibers form in new orientations due to changes in loading. Both isotropic and anisotropic phases act in parallel with the cytoskeleton matrix so that $\boldsymbol{F}_{a}=\boldsymbol{F}_{a}^{i s o}=\boldsymbol{F}_{a}^{a n i}$.

The bio-chemo-mechanically driven re-orientation of the stress-fibers due to external loading is taken into account as follows. In equilibrium, fibers are assumed to align with the maximum principal stretch direction, similar to Menzel[37 and Kuhl et al. ${ }^{[38}$ in the context of the re-orientation of anisotropic biological tissue due to growth and application of loads. The rate of 


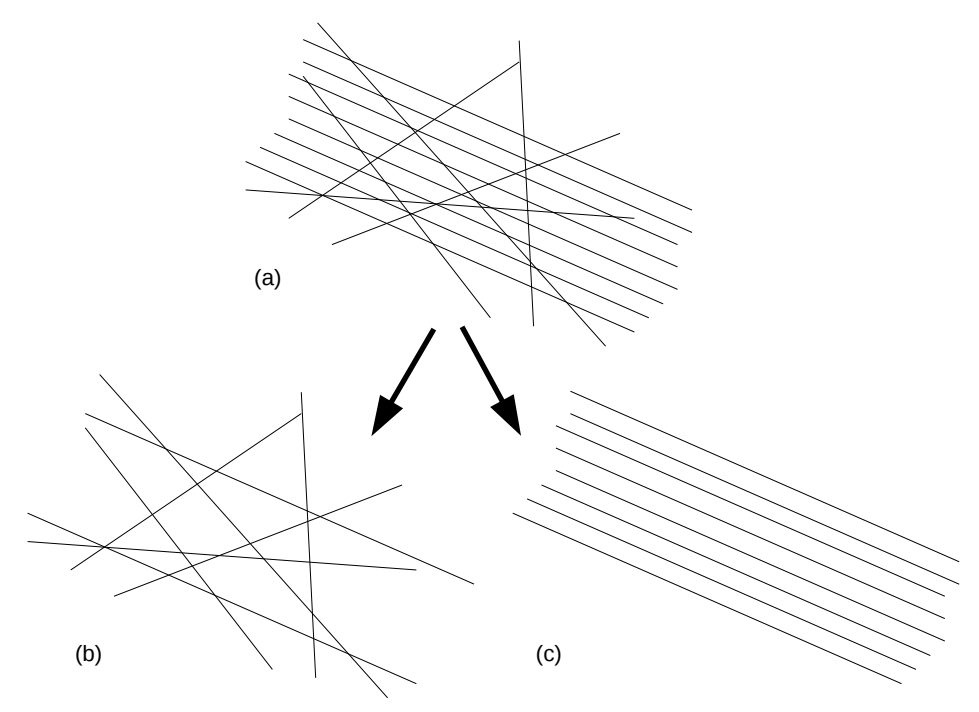

FIGURE 2 2D Schematic of the distribution of fibers (a) and the split into an isotropic (b) and an anisotropic (c) phase

change of the fiber direction vector $\dot{\boldsymbol{n}}_{f i b}$ is then connected to an angular velocity of the average stress fibers through $\boldsymbol{\omega}_{f i b}$

$$
\dot{\boldsymbol{n}}_{f i b}=\boldsymbol{\omega}_{f i b} \times \boldsymbol{n}_{f i b} .
$$

adopting a continuous version of the law presented in [37],

$$
\boldsymbol{\omega}_{f i b}=\frac{2}{\pi \tau} \arctan \left(\frac{\lambda_{1}-\lambda_{2}}{\Delta \lambda_{\text {rot }}}\right) \boldsymbol{n}_{f i b} \times \boldsymbol{n}_{\lambda_{1}}
$$

where the principal stretches $\lambda_{i}$ are ordered as $\lambda_{1} \geq \lambda_{2} \geq \lambda_{3}, \boldsymbol{n}_{\text {fib }}$ is the unit vector in the stress fiber orientation independent of direction and $\boldsymbol{n}_{\lambda_{1}}$ the unit vector in the the maximum principal stretch orientation in the reference state defined through the right Cauchy-Green deformation gradient as

$$
\boldsymbol{C}=\boldsymbol{F}^{T} \cdot \boldsymbol{F}=\sum_{i=1}^{3} \lambda_{i} \boldsymbol{n}_{\lambda_{i}} \otimes \boldsymbol{n}_{\lambda_{i}}
$$

The law in Eq 10 enforces a very gradual decrease of the stress fiber re-orientation for near-equi-biaxial $\left(\lambda_{1} \approx \lambda_{2}>\lambda_{3}\right)$ and near-volumetric deformation states $\left(\lambda_{1} \approx \lambda_{2} \approx \lambda_{3}\right)$. The principal polarization is defined as $\lambda_{1}-\lambda_{2} \geq 0$ and characterizes the deviation from equi-biaxial and volumetric deformation states, as the smallest principal stretch $\lambda_{3}$ is not used in this definition. The characteristic time for this fiber re-orientation process is denoted as $\tau$, and reflects the internal kinetics of stress-fiber creation/dissolution/rotation, while $\Delta \lambda_{\text {rot }}$ is the characteristic polarization for the re-orientation process, which represents a deviation from a non-polarized state relevant to the gradual decrease of the stress fiber re-orientation. 


\section{3 | A model for stress fiber activation}

The isotropic activation is defined as the active material volume fraction, and the anisotropic activation is defined as the active fiber area. They are both normalized over the maximum volume fraction and maximum fiber area equivalently, defined by the bio-chemistry of the cell. We assume that, in equilibrium, both activation phases are proportional to the concentration of active motors, with the proportionality depending on a function of the principal polarization.

$$
\begin{gathered}
\eta_{e q}^{i s o}=f^{i s o}\left(\lambda_{1}^{e q}, \lambda_{2}^{e q}\right) \tilde{c}_{a m}^{e q} \\
\eta_{e q}^{a n i}=f^{a n i}\left(\lambda_{1}^{e q}, \lambda_{2}^{e q}\right) \tilde{c}_{a m}^{e q} .
\end{gathered}
$$

There is no need to introduce any additional constant relating $\eta^{i s o}, \eta^{a n i}$ and $\tilde{c}_{a m}^{e q}$ because it can be absorbed into the subsequent formulation of the free energy functional (see below). The reduced chemical kinetics model of Eq. 7 is employed to follow the concentration of active acto-myosin motors at equilibrium and in the transient regime. The isotropic and anisotropic functions of polarization are taken as

$$
f^{i s o}\left(\lambda_{1}, \lambda_{2}\right)=1-\frac{2}{\pi} \arctan \left(\frac{\lambda_{1}-\lambda_{2}}{\Delta \lambda_{\text {act }}}\right)
$$

and

$$
f^{a n i}\left(\lambda_{1}, \lambda_{2}\right)=\frac{2}{\pi} \arctan \left(\frac{\lambda_{1}-\lambda_{2}}{\Delta \lambda_{\text {act }}}\right)
$$

The sigmoidal function of the principal polarization leads to preferential aligned anisotropic stress-fibers in a highly polarized state through the increase of the anisotropic activation and preferential isotropic stress-fiber network low polarized state (near equi-biaxial or volumetric deformation). A non-dimensional parameter $\Delta \lambda_{\text {act }}$ is introduced to regulate the effect of polarization in the transition from an isotropic to an anisotropic state and vise-versa.

At every instance of the process, a projected equilibrium state can be estimated based on Eq. 12/13 using the current values of the concentration of active motors and polarization (considering $\lambda_{1}^{e q}=\lambda_{1}, \lambda_{2}^{e q}=\lambda_{2}, \tilde{c}_{a m}^{e q}=\tilde{c}_{a m}$ ). The rates of change of activation are assumed to depend linearly on the current deviation from a projected equilibrium state as

$$
\dot{\eta}^{i s o}=r^{i s o}\left(f^{i s o} \tilde{c}_{a m}-\eta^{i s o}\right)
$$

for the isotropic activation and

$$
\dot{\eta}^{a n i}=r^{a n i}\left(f^{a n i} \tilde{c}_{a m}-\eta^{a n i}\right)
$$

for the anisotropic activation. The rate constants $r^{i s o}$ and $r^{a n i}$ capture the time scales of transient evolution of the stress-fibers, and which are presumably on the same order as the time scale $\tau$ for stress-fiber re-orientation. 


\section{4 | The intracellular signaling process}

To recapitulate mechanotransduction, external mechanical stimuli must be converted to the bio-chemical signals that regulate the formation and contraction of stress fibers. The conformational changes of the focal adhesion complexes through mechanical forces are transmitted from the bonded $\beta$-integrins recruiting vinculin, talin, paxilin and focal adhesion kinase to activate GEFs and GAPs, which in turn activate Rho GTPases. RhoA activity promotes polymerization of F-actin and, through ROCK, leads to the activation of myosin. The surface tractions are the combined mechanical forces transfered by individual integrins, and so are the mechanical quantities that determine the signaling. We assume a constant areal density of integrins and propose a linear law to relate the rate of inactive to active myosin transformation to the surface tractions as

$$
k_{1}=k_{0}+\frac{\alpha}{V_{0}} \int_{\partial S} \arctan \left(\frac{H(\boldsymbol{T} \cdot \boldsymbol{n})}{T_{0}}\right) d S
$$

where $k_{0}$ is the base rate at the initial state where no focal adhesions are formed and no external tractions are acting as mentioned in Section 2.1. This directly allows for the calculation of the concentration of active (and inactive) myosin molecules at the initial state, by applying a steady state solution of Eq.7f for the case of no applied tractions. In the last term of Eq 18 $\alpha / V_{0}$ represents the rate of increase of myosin activation in the volume of the cell due to tractions acting over the surface, which are taken into account through the integral over the surface. The Heaviside function acts on the normal tractions to only take into account the positive (tensile) contributions, since the compressive normal traction contributions do not induce any conformational changes to the focal adhesions necessary for intracellular signaling. The reference traction $T_{0}$ is the characteristic traction needed to induce conformational changes in focal adhesions. Currently only normal tractions are considered but shear tractions could also be introduced to the model to control the activation rates of myosin extending this simple signaling approach.

\section{3 | A CONSTITUTIVE MODEL FOR CELL CONTRACTILITY}

\section{1 | Free energy density function}

Recall that we assume that the passive elasticity can be decoupled from the active response of the cell material. The active material is further divided into isotropic and anisotropic components that are also decoupled. With this background, the free energy density of the cell, which was first introduced in Appendix A, can be decomposed as

$$
W\left(\boldsymbol{C}, \eta^{i s o}, \eta^{a n i}, \boldsymbol{n}_{f i b}\right)=W_{p}(\boldsymbol{C})+W_{a}^{i s o}\left(\boldsymbol{C}, \eta^{i s o}\right)+W_{a}^{a n i}\left(\boldsymbol{C}, \eta^{a n i}, \boldsymbol{n}_{f i b}\right)
$$

where $W_{p}$ corresponds to the free energy related to the passive elasticity, $W_{a}^{i s o}$ to the active isotropic stress-fibers, and $W_{a}^{a n i}$ to the the active anisotropic stress fibers. 
The passive mechanical phase is represented as a compressible Neo-Hookean model to model the hyperelastic response of the cytoskeleton

$$
W_{p}(\boldsymbol{C})=W_{e l}\left(I_{1}, J\right)=\frac{\mu}{2}\left(I_{1}-3-2 \ln J\right)+\frac{\beta}{2}(\ln J)^{2}
$$

where $I_{1}=\operatorname{tr}(\boldsymbol{C})$ is the first principal invariant of the right Cauchy-Green deformation gradient tensor, $J=\operatorname{det}(\boldsymbol{F})$ is the third principal invariant related to volumes, $\mu$ is the shear modulus, and $\beta$ is the first Lame parameter (usually denoted by $\lambda$, but changed here to avoid confusion with principal stretches). This model could later be enhanced to a full poroelastic model to account for the effects of cytosol on the elastic response of the cell.

The active isotropic interaction free energy is then taken to be

$$
W_{a}^{i s o}\left(\boldsymbol{C}, \eta^{i s o}\right)=\gamma^{i s o} \eta^{i s o} J^{2}
$$

where $\gamma^{i s o}$ is a chemo-mechanical contraction modulus. Similarly, the active anisotropic part is formulated as

$$
W_{a}^{a n i}\left(\boldsymbol{C}, \eta^{a n i}, \boldsymbol{n}_{f i b}\right)=\gamma^{a n i} \eta^{a n i} I_{4}^{2}
$$

where $\gamma^{a n i}$ is a chemo-mechanical contraction modulus. The invariant $I_{4}$ incorporates the information about the fiber orientation (independent of direction) and the deformation state, and is defined as

$$
I_{4}=\boldsymbol{n}_{f i b} \cdot \boldsymbol{C} \boldsymbol{n}_{f i b} .
$$

which imparts a transversely isotropic character to the active anisotropic part of the free energy. The total free energy of Eq. 19 satisfies the requirement that it should be equal to zero in the reference state. This is enforced as the isotropic and anisotropic activations are both equal to zero in the reference state, eliminating the contributions from Eqs. 21 and 22 in the total energy.

\section{2 $\mid$ Constitutive equations for stress}

Using the balance of internal and external virtual power ${ }^{[39}$ under quasi-static isothermal conditions, a set of balance laws are obtained (see Appendix A) and the standard equilibrium equation follows in Eq. A4 naturally taking into account active and passive stresses. In Appendix A a dissipation inequality is formed and leads to a set of constitutive relationships that have to be enforced for the satisfaction of the inequality. By assumption, there is no spatial gradient with respect to the chemical kinetics of the model. The only constitutive relationship that will be useful for the rest of this work follows from the first parenthesis in the left hand side of Eq. A8 and defines a relationship that holds between the 1st P-K stress and the free energy density as

$$
\boldsymbol{P}=\frac{\partial W}{\partial \boldsymbol{F}}
$$

containing contributions from passive and active elasticity. 
A formula for the 1st P-K stress can be obtained from Eq.24 using the specific form of the free energy given from Eqs. 19.22 It is naturally decomposed into three components as

$$
\boldsymbol{P}=\frac{\partial W}{\partial \boldsymbol{F}}=\frac{\partial W_{p}}{\partial \boldsymbol{F}}+\frac{\partial W_{a}^{i s o}}{\partial \boldsymbol{F}}+\frac{\partial W_{a}^{a n i}}{\partial \boldsymbol{F}}=\boldsymbol{P}_{p}+\boldsymbol{P}_{a}^{i s o}+\boldsymbol{P}_{a}^{a n i}
$$

The passive elastic part of the 1st P-K stress is obtained through

$$
\boldsymbol{P}_{p}=\frac{\partial W_{p}}{\partial \boldsymbol{F}}=\frac{\mu}{2}\left(\frac{\partial I_{1}}{\partial \boldsymbol{C}}: \frac{\partial \boldsymbol{C}}{\partial \boldsymbol{F}}-\frac{2}{J} \frac{\partial J}{\partial \boldsymbol{F}}\right)+\beta \frac{\ln J}{J} \frac{\partial J}{\partial \boldsymbol{F}}
$$

The following derivatives of the invariants

$$
\frac{\partial J}{\partial \boldsymbol{F}}=J \boldsymbol{F}^{-T}, \quad \frac{\partial I_{1}}{\partial \boldsymbol{C}}=\boldsymbol{I}
$$

are used in Eq. 26 and the following relationship holds

$$
\frac{\partial()}{\partial \boldsymbol{F}}=\frac{\partial()}{\partial \boldsymbol{C}}: \frac{\partial \boldsymbol{C}}{\partial \boldsymbol{F}}=2 \boldsymbol{F} \cdot \frac{\partial()}{\partial \boldsymbol{C}}
$$

For the isotropic active part of the 1st P-K stress it holds that

$$
\boldsymbol{P}_{a}^{i s o}=\frac{\partial W_{a}^{i s o}}{\partial \boldsymbol{F}}=2 \gamma^{i s o} \eta^{i s o} \boldsymbol{J}^{2} \boldsymbol{F}^{-T}
$$

Applying the chain rule, the active anisotropic contractile part of the 1st P-K then follows

$$
\boldsymbol{P}_{a}^{a n i}=\frac{\partial W_{a}^{a n i}}{\partial \boldsymbol{F}}=2 \gamma^{a n i} \eta^{a n i} I_{4} \frac{\partial I_{4}}{\partial \boldsymbol{F}}
$$

where the derivative of the invariant $I_{4}$ with respect to the right Cauchy-Green deformation gradient is given through

$$
\frac{\partial I_{4}}{\partial \boldsymbol{C}}=\boldsymbol{n}_{f i b} \otimes \boldsymbol{n}_{f i b}
$$

Combining Eqs 25 31 leads to a complete expression for the 1st P-K stress tensor. Tangent moduli are calculated in Appendix B.

\section{3 | Material parameters}

We now select values of the various material properties that appear in the material model summarized in Table 1 . These values are used for the remainder of this work unless otherwise noted. We set the ratio of Lame's first constant to shear modulus to $\beta / \mu=1$, which allows significant compressibility in the material, necessary to mimic significant cell volume changes due to poroelastic effects. The isotropic and anisotropic contraction moduli are set to $\gamma^{i s o} / \mu=1$ and $\gamma^{a n i} / \mu=1$, since the stresses that develop due to the stress fibers should be of the same order of magnitude as the stresses in the passive network at similar deformation states and supportable by the forces transferred through the focal adhesions. The reference traction for the signaling 
law is also taken as $T_{0} / \mu=1$ since the tractions necessary to induce conformational changes at the focal adhesions and increase of RhoA activity are expected to be on the same order of magnitude as the contraction moduli.

\begin{tabular}{|l|l|}
\hline$\mu$ & Material properties \\
$\beta$ & Lame's first constant \\
$\gamma^{\text {iso }}$ & isotropic contraction modulus \\
$\gamma^{\text {ani }}$ & anisotropic contraction modulus \\
$T_{0}$ & reference cell surface traction \\
$k_{0}$ & base rate of myosin activation \\
$k_{2}$ & rate of myosin de-activation \\
$\tau$ & characteristic time for stress fiber re-orientation \\
$r^{i s o}$ & rate constant for isotropic activation \\
$r^{\text {ani }}$ & rate constant for anisotropic activation \\
$\alpha$ & signaling constant \\
$\Delta \lambda_{\text {rot }}$ & characteristic rotation rate polarization \\
$\Delta \lambda_{\text {act }}$ & characteristic activation polarization \\
\hline
\end{tabular}

TABLE 1 List of material parameters used in the proposed cellular mechanotransduction and contractility model

We normalize all time quantities with respect to the base rate $k_{0}$ for transformation of inactive myosin to active myosin in the free homeostatic state. For the additional chemical kinetics of myosin, we set $k_{2} / k_{0}=5$ so that the homeostatic state has a low concentration of active myosin (from steady state solution of Eq. 7). The normalized characteristic time for anisotropic stress-fiber re-orientation is taken to be $\tau=0.1 / k_{0}$ while the rates constants for isotropic and anisotropic activation are taken as $r^{i s o} / k_{0}=r^{a n i} / k_{0}=10$, corresponding to the same time scale as the re-orientation process. The traction-controlled signaling has a constant $\alpha /\left(V_{0}^{1 / 3} k_{0}\right)=10$.

The effect of polarization on the rotation rate of the average anisotropic stress fiber direction is realized setting the characteristic rotation rate polarization to be $\Delta \lambda_{\text {rot }}=0.05$ so that the reorientation process is very slow at low polarizations. The characteristic activation polarization (equal for isotropic and anisotropic activation) is taken to be $\Delta \lambda_{\text {act }}=1$ so that stress-fiber alignment occurs at polarization levels comparable to those observed experimentally 40$]$ [1].

In principle, all the material constants should be calibrated to experiments to achieve an accurate predictive model. At the present time, this is not possible because the model does not include an active model for focal adhesions. We are not aware of any experimental studies in which the effects of internal cell evolution and focal adhesion are not both active, the latter driving the 
creation of tractions on the cell boundaries which in turn activates the internal contractility and stress-fiber development. Once a focal adhesion model is developed, meaningful experimental comparisons will be possible. Nonetheless, the present model is necessary to establish a realistic interplay of the various features of internal cell evolution (stress fiber formation/dissociation/reorientation) due to an applied complex load path. The current choice of material parameters are physically reasonable and enable demonstration of important features of the cell deformation and evolution processes, as presented in the next section. The parameters used in the proposed model are summarized in Table 1

\section{4 | EXERCISING THE MODEL: RESULTS}

The numerical implementation of the model using a finite element method is discussed in Appendix C. This allows for the numerical solution of benchmark initial boundary value problems in order to demonstrate the behaviors that emerge from the model. All benchmark problems are solved in a 3D setting, but we categorize them as 2D and 3D problems depending on the complexity of the response.

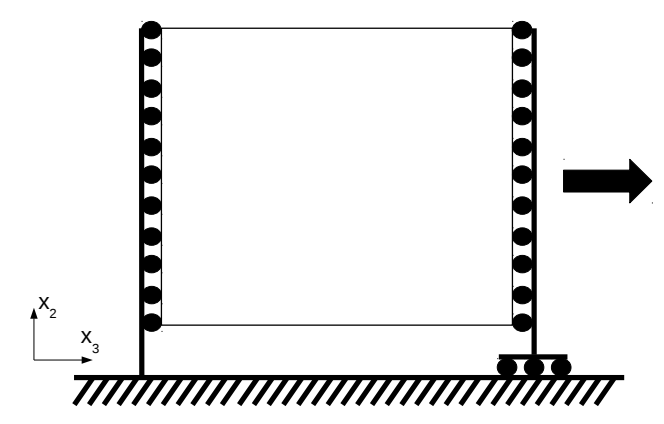

FIGURE 3 Schematic of the force controlled stage of the 2-stage loading of a single cell

\section{1 | 2-stage loading in 2D}

First, a 2D 2-stage loading scenario is studied, where a cell is instantaneously loaded (under both force and displacement control) and then unloaded, to validate that once the cell contracts, it will eventually return to its initial configuration upon release of the external load. The 2-stage loading test is motivated by the type of loading experienced by a contractile cell during damage of a matrix in which it is embedded ${ }^{[42]}$ or when a cell that has contracted due to contact with a substrate is released to a free state ${ }^{[43]}$. In the former case, the matrix is loaded under displacement or force control, which leads to contraction of the cells through mechanotransduction. As damage is induced, the contractile cell experiences a sudden change in external loading, which again 
(a)

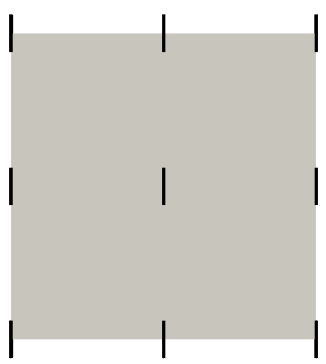

(d)

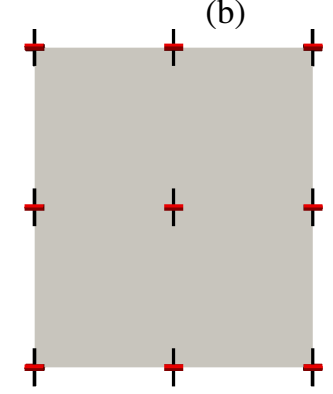

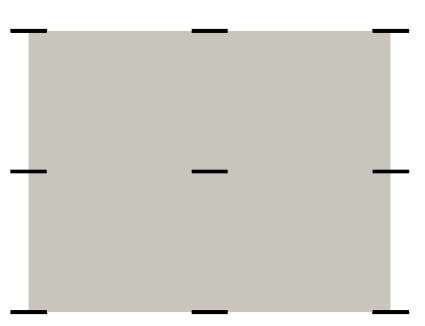

(c)
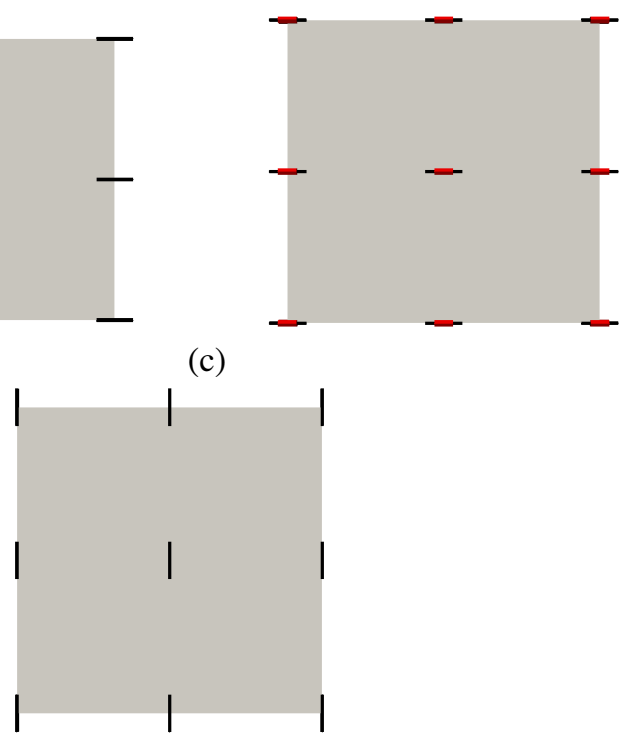

(e)

FIGURE 4 Instances of the 2-stage loading case, under force control. The black lines are aligned with the orientation of the maximum principal stretch. The red lines are aligned with the anisotropic average fiber orientation, and their width scales with the anisotropic activation

signals the cell to change its contractile behavior. More often, cells experience continuous changes of external loading as the mechano-transduction mechanism is influenced by the evolution of the focal adhesions on the cell boundary. The 2-stage loading here is thus crucial to showcase the characteristics of the active material model and signaling model in an environment where the external loading changes.

A cubic geometry of reference edge length $L$ is considered for the single cell. We apply the same type of loading cycle as experienced by a cell under certain experimental conditions as discussed above. For the 1 st stage of the loading, a set of boundary conditions in imposed at $t=0^{+}$and maintained until $t=t_{0}^{+}$. The end at $X_{3}=0$ is fixed in the $X_{3}$ direction so that $\left.u_{3}\right|_{X_{3}=0}=0$, and the end at $X_{3}=L$ is constrained so that it remains parallel to the $X_{1}, X_{2}$ plane with $\left.u_{3}\right|_{X_{3}=L}=\bar{u}_{3}(t)$. A constant load $f_{0}=\beta_{1} L^{2}$ is applied in the $x_{3}$ direction. A schematic for this loading stage is shown in Fig. 3 This initiates the intracellular signaling mechanism, which leads to a build-up of the contractility. Consequently, the applied load and the displacement constraints are are removed at $t=t_{0}^{+}$and the cell remains traction free and free of displacement constraints. Upon release of the load, the cell instantaneously contracts, but the contractility decreases only gradually and eventually the cell returns to its initial free state. As discussed in ${ }^{19}$, activated stress fibers dissociate after removal of externally applied loads and cells return to their initial state without signs of residual stresses. The initial values of the concentration of active motors is calculated from Eq. 7 setting $\dot{\tilde{c}}_{a m}=0$ and $k_{1}=k_{0}$. The initial values of the isotropic and the anisotropic activation are calculated directly from Eq. 12 and 13 with $\left(f^{i s o}\right)^{0}=1$ and $\left(f^{i s o}\right)^{0}=0$ since the initial state is not polarized. The initial value of stretch $\lambda^{\text {init }}$ corresponding to the homogeneous deformation of the initial free homeostatic state is given by Eq. D17 For simplicity, the initial direction of the 

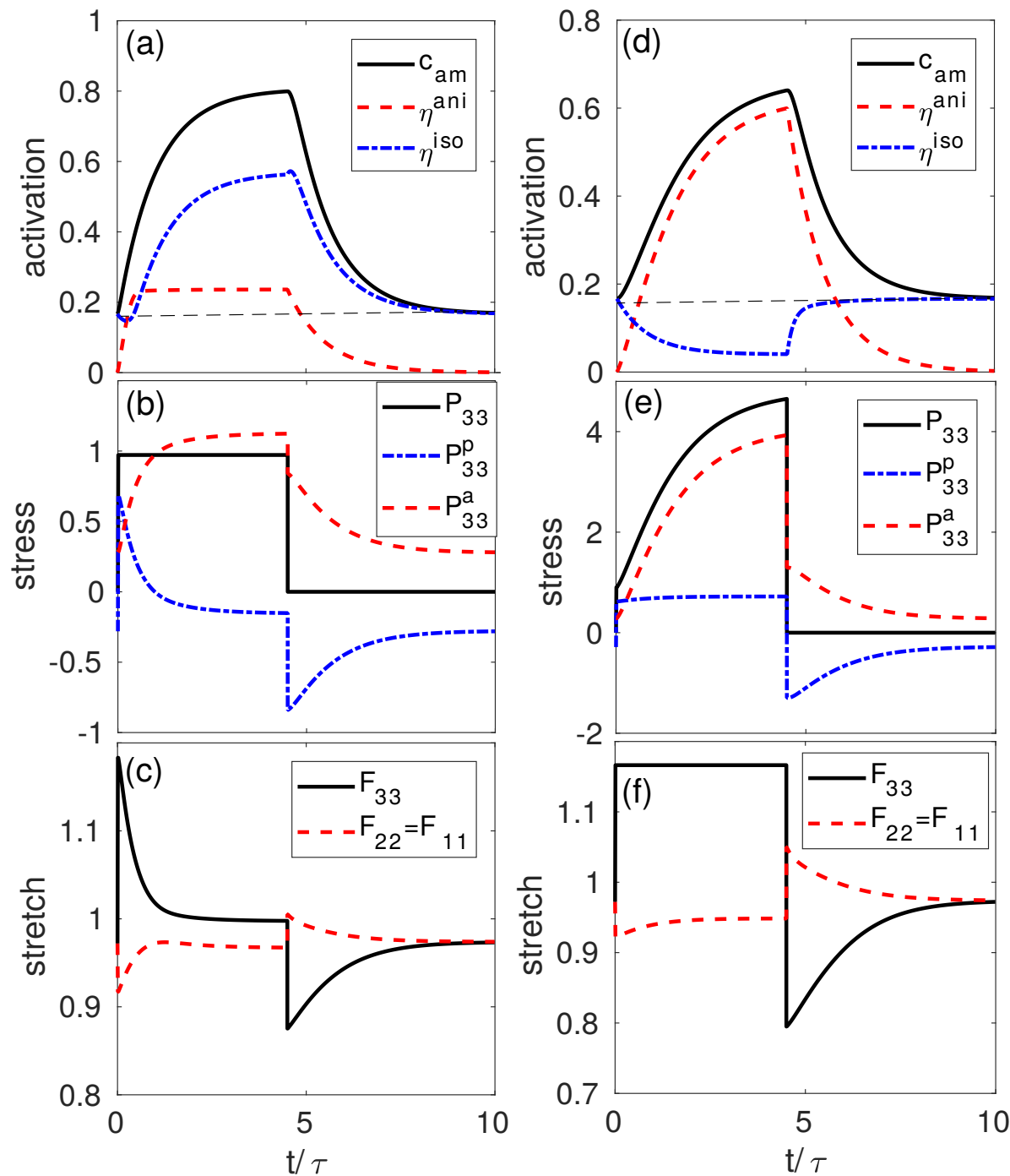

FIGURE 5 Results of 2-stage loading under force control (a-c) and displacement control (d-f), for the evolution of the activation (a)(d), normalized stress (b)(e) and stretch(c)(f)

fibers $\boldsymbol{n}_{f i b}^{0}$, which is required even at zero anisotropic activation, is taken to be aligned with the $X_{3}$ axis $\boldsymbol{n} f i b^{0}=\boldsymbol{n}_{3}$ everywhere throughout the body, but a random distribution of directions could also be chosen. Unloading occurs at $t_{0} / \tau=4.5$.

The time evolution of the system and various quantities is shown in 4 , where state (a) is the initial homogeneously contracted state, state (b) is the instantaneously loaded state, (c) is the contracted state of the cell with the constraints and load still in place at $t=t_{0}^{-}$just before the load is released, state (d) is the equi-biaxially contracted state at $t=t_{0}^{+}$just after release of the constraints, and state (e) is the final equilibrated state that is the same as the initial state. The black lines are aligned with the orientation of the maximum principal stretch $\boldsymbol{n}_{\lambda_{1}}$. The the red line are aligned with the average fiber orientation $\boldsymbol{n}_{f i b}$, and the width of the red lines is proportional to the magnitude of the anisotropic activation $\eta^{\text {ani }}$. Fig 5 compares results under force-control (Fig 5 a-c) and displacement-control (Fig 5 d-f) for the evolution of the activation(Fig 5 a,d), the stress(Fig 5 b,e) and the stretch (Fig 5 c,f). Under force control, the total stress is fixed until $t_{0}$ but, as seen in 5 b), upon unloading there is an abrupt jump in 
the passive stress. This jump corresponds to the state shown in 4 a), where anisotropic activation induces an active stress that leads to a state of biaxial compression, whereas the final deformation state 4 e) is isotropic. In this case, because the cell can contract in the $X_{3}$ direction starting at $t=0$, the isotropic activation is more prominent than the anisotropic activation, as seen in 5 a), because the polarization decreasses after application of the load at $t_{0}$, as seen in 5 f).
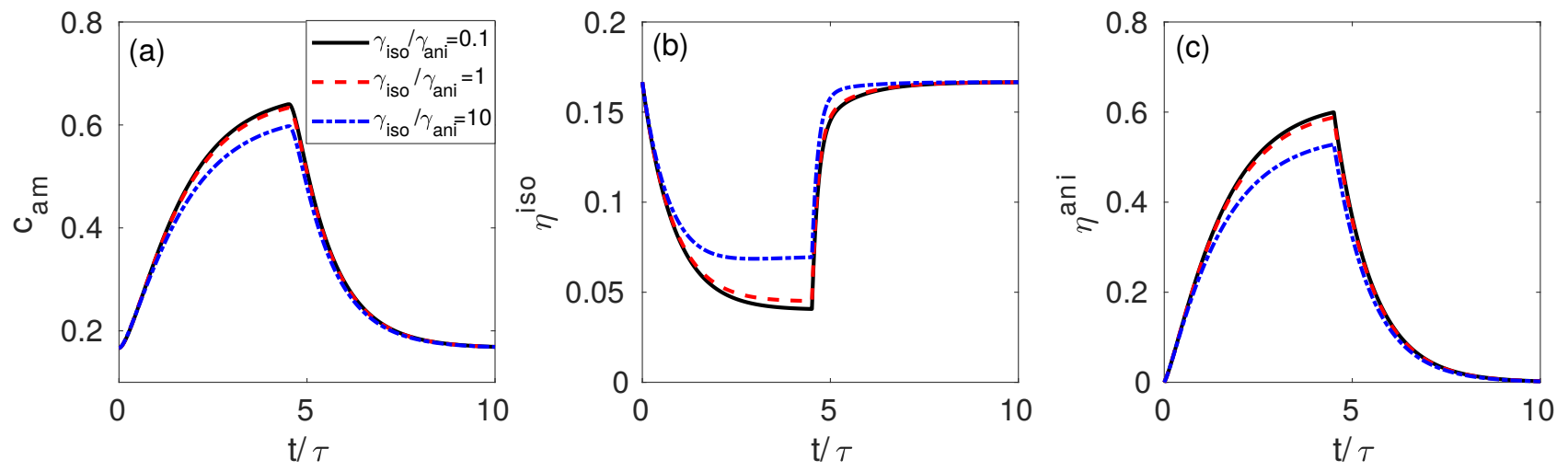

FIGURE 62 -stage loading under displacement-control for different contraction moduli ratios $\gamma^{i s o} / \gamma^{a n i}=0.1,1,10$. Evolution of the active myosin concentration (a), the isotropic (b) and the anisotropic activation (c)
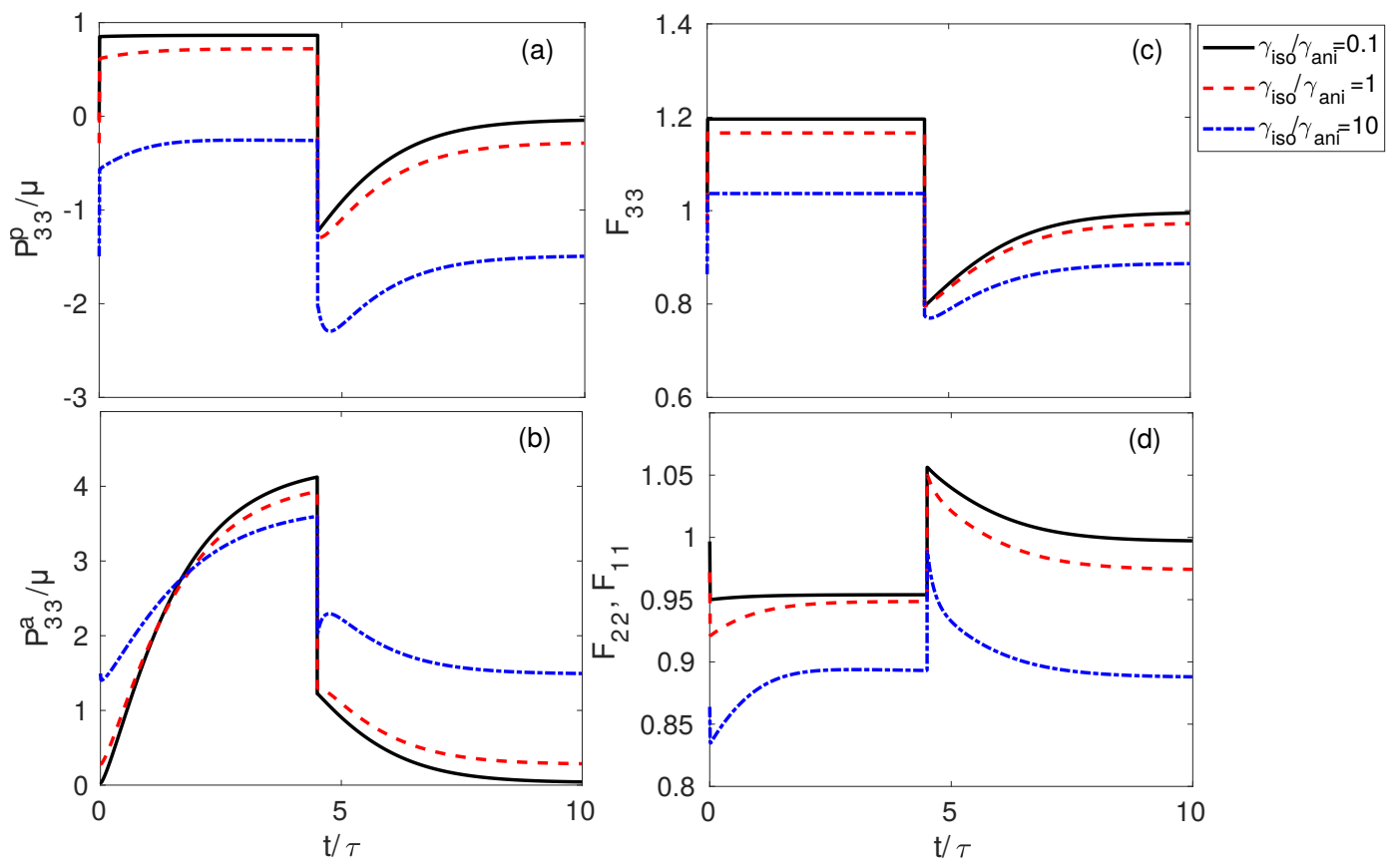

FIGURE 72 -stage loading under displacement-control for different contraction moduli ratios $\gamma^{i s o} / \gamma^{a n i}=0.1,1,10$. Evolution of the passive normalized stress (a), active normalized stress (b) the stretch in the loading direction (c) and the stretch perpendicular for the loading direction (d). 
As a second test a similar loading cycle is considered, but under displacement control for the first stage. The same cell geometry is considered. For the first stage of the loading, a set of boundary conditions in imposed at $t=0^{+}$and maintained until $t=t_{0}^{+}$. The end at $X_{3}=0$ is fixed in the $X_{3}$ direction so that $\left.u_{3}\right|_{X_{3}=0}=0$, and the end at $X_{3}=L$ is constrained in the $X_{3}$ direction with $\left.u_{3}\right|_{X_{3}=L}=\bar{u}_{3}$. Specifically, a displacement $\bar{u}_{3}=0.2 \lambda^{i n i t} L$ is applied on that face. The applied displacement constraints are removed at $t=t_{0}^{+}$and the cell remains traction free. The same initial conditions and loading duration are used as in the load controlled case. In Fig. 5 (d) the evolution of the activation components and the concentration of active myosin are shown. The concentration of active myosin increases until the cell is unloaded and then returns to its initial state. Being under displacement control, $F_{33}$ is fixed (becoming the maximum principal stretch $\lambda_{1}$ ) until $t_{0}$ while the other principal stretches diminish, as seen in 5 f). This leads to an increase of the polarization during the first loading phase. The anisotropic activation thus increases while the isotropic activation decreases. Upon release of the constraints at $t_{0}^{+}$, the cell is under equi-biaxial compression leading to slowing down of the re-orientation of the anisotropic stress fiber distribution. Eventually both activation components relax to their initial values at equilibrium, where the cell is not polarized. In Fig. 5 e), the total stress in the $X_{3}$ direction increases when the displacement is imposed, and then relaxes to zero upon the release of the constraints. The initial increase is induced as the active part of the stress increases in $X_{3}$ direction. In the unloaded homeostatic state, the active and passive stress become equal and opposite, as expected.

In Figs. 6 6 7 we examine the role of the relative magnitudes of the anisotropic contraction modulus $\gamma^{\text {ani }}$ and the isotropic modulus $\gamma^{i s o}$ for 2-stage loading under displacement control with the same initial conditions, boundary conditions and loading duration as the previous example under displacement control. We consider $\gamma^{\text {iso }} / \gamma^{\text {ani }}=0.1,1,10$ holding $\gamma^{\text {ani }}=1$ fixed.The initial contraction is more intense for the cases of high ratio leading to smaller $\lambda^{\text {init }}$. Fig. 6 shows the evolution of the concentration of active myosin (a) and the activation evolution $(b, c)$ for the different moduli ratios showing a more rapid increase for the active myosin concentration and the isotropic and anisotropic activation for lower contraction moduli ratios. This result effectively demonstrates the Poisson's effect induced by high isotropic contraction moduli during the contraction process, having a significant effect on the intracellular signaling process. Fig. 7 shows the evolution of the passive stress (a), active stress (b), stretch in the loading direction (c), and stretch perpendicular to the loading direction (d). The short term increase of the active stress Fig. 7 (b) upon release of the load in the high ratio case is due to the rapid increase of the isotropic activation, as seen in Fig. 6 (b), and the decrease of the anisotropic activation, as seen in Fig. 6 (c). The initial increase of the $F_{22}=F_{11}$ stretch (Fig. 6 (c)) is due to the decrease of the isotropic activation upon initial polarization of the specimen, and is more prominent for high moduli ratios. This variation of the isotropic and anisotropic contraction moduli ratio allows us to observe complicated response patterns, when the ratio is high. Being able to follow the stretch experimentally as in Fig. 6 .c-d) will eventually allow for the calibration of the contraction moduli, or at least for a meaningful interpretation of the experimental observations with respect to the proposed model. 


\section{2 | 3D contraction}

(a)

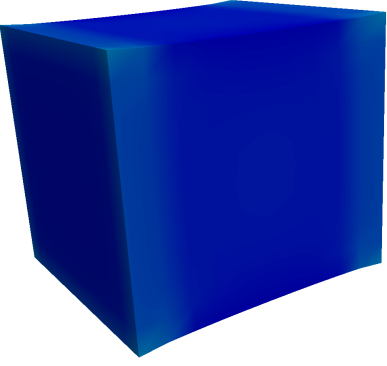

(b)

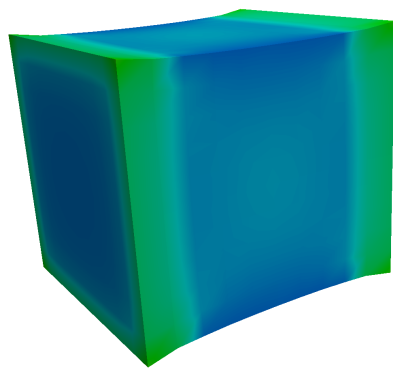

(c)

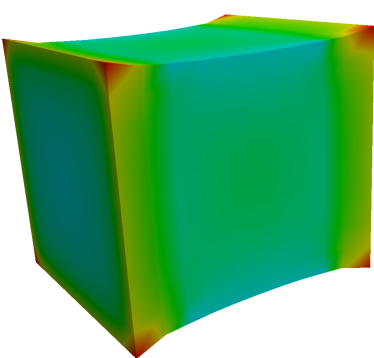

(d)

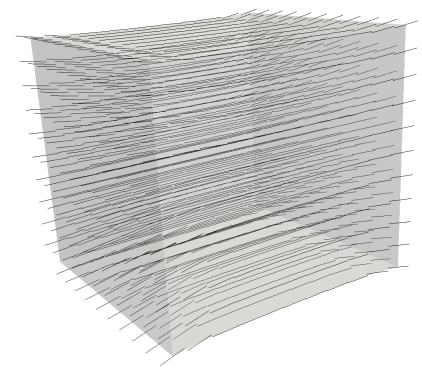

(e)

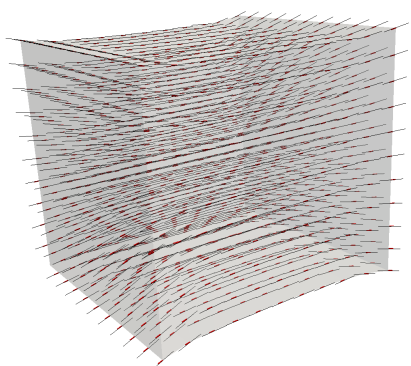

(f)

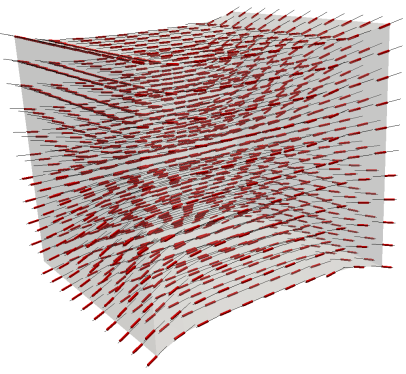

FIGURE 8 For uniaxial extension of a single contractile cell, with fixed displacement on the clamped ends, contour plot of the $P_{33}$ normalized stress at times $t / \tau=0^{+}, 1,100$ shown in (a) (b) (c) which correspond to (a) the instantaneous response once external loading is applied, (b) an intermediate state where stress-fiber alignment and re-orientation is observed, and (c) the equilibrium state where stress-fiber formation and dissociation have reached a steady state. The stress-fiber realignment process is demonstrated in (d) (e) (f) at the same instances in time .

To demonstrate the full capabilities of the cell model including the stress-fiber reorientation, we perform a 3D simulation that exhibits inhomogeneous deformations. Again a cubic geometry is considered. The face of the cube at $X_{3}=0$ is held fixed with $\left.u_{i}\right|_{X_{3}=0}=0(i=1,3)$, and the face at $X_{3}=L$ is clamped $\left.u_{1}\right|_{X_{3}=L}=\left.u_{2}\right|_{X_{3}=L}=0$ and is displaced in the $X_{3}$ direction by $\left.u_{3}\right|_{X_{3}=L}=\bar{u}_{3}$, with $\bar{u}_{3}=0.1 \lambda^{\text {init }} L$ and held fixed. Geometry, material properties and initial conditions the same as those for the 2D 2-stage study in Section 4.1.

The response of the system after displacement is imposed, $t>0$, tractions develop on the clamped surfaces and these drive increased activation. The cell contracts until an equilibrium state is obtained. As the activation level increases, the reaction force also increases as the contractile forces develop. An inhomogeneous deformation field develops, with the the fibers aligning with the orientation of the local principal stretches. In Figs. 8 (a) (b) (c) corresponding 1st P-K stress fields for the complete 3D 
structure are shown at times $t / \tau=0^{+}, 1,100$, showing the intensifying stress during contraction. Figs. 8 (d) (e) (f) again at $t / \tau=0^{+}, 1,100$, where the black lines are aligned the orientation of the maximum principal stretch $\boldsymbol{n}_{\lambda_{1}}$, the red lines are aligned with the local anisotropic stress-fiber orientation $\boldsymbol{n}_{f i b}$, and the width of the lines is proportional to the anisotropic activation $\eta^{a} n i$. Even though cellular adhesion is not modeled explicitly, the response that the model captures in this numerical experiment is similar to the physical response of single cells, predicting contractile response and stress fiber formation and re-orientation as a response to an external mechanical stimulus. This is a critical feature towards building a complete model for cell response including focal adhesions, as it shows that boundary conditions can influence the direction of stress fibers that have formed.

\section{5 | DISCUSSION}

A physically-motivated model for cell contraction, mechanosensing and signal transduction is proposed. The active material model includes passive elasticity recapitulating the properties of the cytoskeleton and isotropic and anisotropic phases for the stress-fiber distribution. The activation is controlled by myosin chemical kinetics and polymerization of F-actin through a reduced version of the Hai-Murphy model. Signal processing is introduced through a law that connects the normal tractions on the cell surface with the intracellular RhoA signaling mechanism that leads to changes in the rate of of activation of myosin and polymerization of F-actin. This leads to a cooperative signaling mechanism that transfers information about the tractions on the cell surface into the active material model and also gets feedback from the cell depending on the mechanical interactions with its surroundings. A law for the realignment of average stress fibers driven by the maximum principal stretch directions is also considered. This framework allows for a functional mechanism to study the interaction of cells with their surroundings.

Deriving a set of balance laws and obtaining the constitutive relationship used from the dissipation inequality ensures thermodynamic consistency of the model. A free energy density function is assumed to be decomposed into a passive and active component. The stress constitutive relationship gives a combined expression for both components. Tractions on the cell surface influence the level of stress fiber activation through the model of intracellular signaling mechanism proposed, causing a direct effect in the active material response through the active part of the free energy density. Since the thermodynamic framework that the theory was built on allows for the study of other effects, it would be interesting to study additional signaling mechanisms involved in the contractile response of cells. For example, introduction of a continuum model for the formation of focal adhesions and their effect on signaling and contractility can be realized through modulating the tractions on the cell surface. That would allow simulation of cell response in real-time as a result of the cell-ECM interactions. The model can also be extended to capture poroelastic effects along with the contractile behavior of the cell. Applying this model to study tissues in a physiological and pathological context is possible once an appropriate model for the interaction of cells with their surroundings is in place. 
Towards this goal, development of a coarse-grained tissue model maintaining the signaling mechanism devised for single cells is another direction that remains to be pursued.

How cite this article: N. Bouklas, M.S. Sakar, and W.A. Curtin (****), A model for cellular mechanotransduction and contractility at finite strain, $* * * * *$.

\section{APPENDIX A: BALANCE LAWS AND DISSIPATION INEQUALITY}

Through the principle of virtual power ${ }^{39]}$ [29], a set of balance laws are derived for the contractile behavior of single cells and the processes including stress fiber activation and re-orientation. The fluxes that are relevant to the change of state in this problem are the following: a) a set of admissible velocities $\mathcal{V}$, b) a set of admissible rates of activation of the isotropic and anisotropic mechanisms that acto-myosin motors act through, labeled as $\mathcal{R}_{1}=\mathcal{R}^{i s o}$ and $\mathcal{R}_{2}=\mathcal{R}^{a n i}$ in the same order they were mentioned, and c) a set of admissible rates of change of orientation of the average fibers $\boldsymbol{\mathcal { R }}_{r}$. Under quasi-static isothermal conditions, the internal $N_{i}\left(\mathcal{V}, \mathcal{R}_{i}, \mathcal{R}_{r}\right)$ and external $N_{e}\left(\mathcal{V}, \mathcal{R}_{i}, \boldsymbol{R}_{r}\right)$ power production must satisfy

$$
N_{i}+N_{e}=0 \text { in } \mathcal{D}
$$

for any part $\mathcal{D} \in \Omega_{0}$ of the body. The internal and external power production can be defined as

$$
\begin{gathered}
N_{i}=-\int_{D}\left(\boldsymbol{P}_{p}+\boldsymbol{P}_{a}^{i s o}+\boldsymbol{P}_{a}^{a n i}\right): \nabla_{X} \mathcal{V} d V \\
-\int_{D} \sum_{i} P_{i}^{a} \mathcal{R}_{i} d V-\int_{D} \mathbf{P}_{\mathbf{r}} \cdot \mathcal{R}_{\mathbf{r}} \mathbf{d V} \\
N_{e}=\int_{j \mathcal{D}} \boldsymbol{T} \cdot \mathcal{V} d V-\int_{D} \sum_{i} T_{i}^{a} \mathcal{R}_{i} d V-\int_{D} \mathbf{T}_{\mathbf{r}} \cdot \mathcal{R}_{\mathbf{r}} \mathbf{d V}
\end{gathered}
$$

where $\boldsymbol{P}=\boldsymbol{P}_{p}+\boldsymbol{P}_{a}^{i s o}+\boldsymbol{P}_{a}^{a n i}$ is the 1st Piola-Kirchhoff stress tensor, equal to the sum of a 1st P-K type passive and active stress tensors, denoted equivalently $\boldsymbol{P}_{p}, \boldsymbol{P}_{a}^{i s o}$ and $\boldsymbol{P}_{a}^{a n i}$, and $\boldsymbol{T}$ is the material traction vector. The internal and external thermodynamic forces that are power conjugates with $\mathcal{R}_{i}$ are $P_{i}^{m}$ and $T_{i}^{m}$, and the ones associated with $\mathcal{R}_{r}$ are $\mathbf{P}_{\mathbf{r}}$ and $\mathbf{T}_{\mathbf{r}}$. Combining Eqs. A1, A3 leads to a set of balance laws. The standard mechanical equilibrium equation follows after application of the divergence theorem

$$
\nabla_{X} \cdot \boldsymbol{P}=\mathbf{0} \text { in } \Omega_{0}, \boldsymbol{T}=\boldsymbol{P}^{\mathbf{T}} \boldsymbol{N} \text { on } \partial \Omega_{0}
$$

and the rest of the balance laws are

$$
P_{i}^{a}=-T_{i}^{a} \text { for } \mathrm{i}=1,2 \text { in } \Omega_{0}
$$




$$
\mathbf{P}_{\mathbf{r}}=-\mathbf{T}_{\mathbf{r}} \text { in } \Omega_{0} .
$$

Considering that part of the internal power is stored and part of it is dissipated, a dissipation inequality is formed as follows

$$
\dot{W} \leq \boldsymbol{P}: \dot{\boldsymbol{F}}+P_{m}^{i s o} \dot{\eta}^{i s o}+P_{m}^{a n i} \dot{\eta}^{a n i}+\boldsymbol{P}_{r} \cdot \dot{\boldsymbol{n}}_{f i b}
$$

where () denotes time differentiation, $W$ is the free energy density. $P_{m}^{i s o}$ and $P_{m}^{a n i}$ are internal thermodynamic forces that are power-conjugate to the rate of change of the isotropic and anisotropic stress-fiber activation $\dot{\eta}^{i s o}, \dot{\eta}^{\text {ani }}$. Equivalently the internal thermodynamic force $\boldsymbol{P}_{r}$ is power conjugate to rate of the change of the fiber direction vector $\dot{\boldsymbol{n}}_{f i b}$ and is related to the biochemical processes leading to the re-orientation. Here the individual pathways of chemical processes are not considered explicitly and only their aggregate effect is considered. The chemical kinetics of myosin are also related to the introduction of energy in the form of ATP to the system allowing it to deviate from minimum energy states.

The state variables of the free energy are postulated to be $W\left(\boldsymbol{C}, \eta^{i s o}, \eta^{a n i}, \boldsymbol{n}_{f i b}\right)$, with $\boldsymbol{C}$ the right Cauchy-Green deformation tensor, the activation of isotropic and aligned in stress-fibers acto-myosin motors $\eta^{i s o}$ and $\eta^{a n i}$, and the orientation of the average stress-fibers $\boldsymbol{n}_{f i b}$. Using Eq. A7 and applying the chain rule leads to

$$
\begin{aligned}
& \left(\boldsymbol{P}-\frac{\partial W}{\partial \boldsymbol{F}}\right): \dot{\boldsymbol{F}}+\left(P_{a}^{i s o}-\frac{\partial W}{\partial c_{a m}}\right) \dot{\eta}^{i s o}+ \\
& \left(P_{a}^{a n i}-\frac{\partial W}{\partial c_{i m}}\right) \dot{\eta}^{a n i}+\left(\mathbf{P}_{\mathbf{r}}-\frac{\partial \mathbf{W}}{\partial \boldsymbol{n}_{\mathbf{f i b}}}\right) \cdot \dot{\boldsymbol{n}}_{f i b} \geq 0
\end{aligned}
$$

which can be satisfied by setting the parentheses individually to equal zero. Following, the governing laws for stress-fiber rotation are allowed to hold and we are able to define activation laws that satisfy the dissipation inequality.

\section{APPENDIX B: TANGENT MODULI}

Analytical expressions of the tangent moduli of the active material model using the specific free energy density function that was introduced in Section 3 are presented in this appendix. The notation that follows for the tangent moduli is

$$
\mathbb{A}=\frac{\partial \boldsymbol{P}}{\partial \boldsymbol{F}}=\frac{\partial \boldsymbol{P}_{p}}{\partial \boldsymbol{F}}+\frac{\partial \boldsymbol{P}_{a}}{\partial \boldsymbol{F}}
$$

The variation of the passive term is given

$$
\frac{\partial \boldsymbol{P}_{p}}{\partial \boldsymbol{F}}=\mu\left[\llbracket \cdot \boldsymbol{I}-\llbracket: \frac{\partial \boldsymbol{F}^{-T}}{\partial \boldsymbol{F}}\right]+\beta\left(\boldsymbol{F}^{-T} \otimes \boldsymbol{F}^{-T}+\ln J \frac{\partial \boldsymbol{F}^{-T}}{\partial \boldsymbol{F}}\right)
$$

where $\square$ is the fourth order identity tensor. The equivalent operation for the active stress gives 


$$
\begin{aligned}
\frac{\partial \boldsymbol{P}_{a}}{\partial \boldsymbol{F}} & =2 \gamma^{i s o} \eta^{i s o}\left(2 \boldsymbol{J} \boldsymbol{F}^{-T} \otimes \frac{\partial J}{\partial \boldsymbol{F}}+J^{2} \frac{\partial \boldsymbol{F}^{-T}}{\partial \boldsymbol{F}}\right) \\
& +4 \gamma^{a n i} \eta^{a n i}\left[\left(2 \boldsymbol{F} \cdot \frac{\partial I_{4}}{\partial \boldsymbol{C}}\right) \otimes\left(\boldsymbol{F} \cdot \frac{\partial I_{4}}{\partial \boldsymbol{C}}\right)+I_{4} \rrbracket \cdot \frac{\partial I_{4}}{\partial \boldsymbol{C}}\right]
\end{aligned}
$$

\section{APPENDIX C: FINITE ELEMENT IMPLEMENTATION}

Eq. A4 forms the complete strong form of the problem, thus the weak form of the problem can be formulated as

$$
\begin{gathered}
\int_{V_{0}} \boldsymbol{P}: \nabla_{X} \delta u d V=\mathbf{0} \text { in } \Omega_{0} \\
\boldsymbol{T}=\boldsymbol{P}^{T} \boldsymbol{N}, \delta \boldsymbol{u}=\mathbf{0} \quad \text { on } \partial \Omega_{0}
\end{gathered}
$$

The weak form should be taken into account along with the chemical kinetics Eq. 7 the rates of isotropic and anisotropic activation from Eq. 16 and 17 and the fiber reorientation Eq.10. We choose explicit schemes for time integration of the previous equations. Secifically, a forward Euler scheme is employed for the chemical kinetics equation and the rates of isotropic and anisotropic activation. Whereas the 3D equation for fiber reorientation is integrated temporally using a variation of an EulerRodriguez exponential integration scheme, and specifically an explicit version as presented in the work by Kuhl et al. 38 .

$$
\begin{aligned}
\boldsymbol{n}_{f i b}^{t+\Delta t} & =\cos \left(\Delta t \omega_{f i b}\right) \boldsymbol{n}_{f i b}^{t}+\sin \left(\Delta t \omega_{f i b}\right) \boldsymbol{n}_{\omega} \times \boldsymbol{n}_{f i b}^{t} \\
& =+\left[1-\cos \left(\Delta t \omega_{f i b}\right)\right]\left[\boldsymbol{n}_{\omega} \cdot \boldsymbol{n}_{f i b}^{t}\right] \boldsymbol{n}_{\omega}
\end{aligned}
$$

where the approximation

$$
\boldsymbol{\omega}_{f i b} \approx \frac{2}{\pi \tau} \arctan \left(\frac{\lambda_{1}^{t}-\lambda_{2}^{t}}{\zeta}\right) \boldsymbol{n}_{f i b}^{t} \times \boldsymbol{n}_{\lambda_{1}}^{t}
$$

is considered. Superscript $t+\Delta t$ refers to the current time step and $t$ to the previous time step. Since we use explicit integration schemes attention to time-step size is critical.

A Newton-Raphson procedure is implemented for the solution of the nonlinear problem that arises from Eq.C12 and C13 For the Newton-Raphson procedure even though we presented some tangent moduli in Appendix B, and Menzel ${ }^{[37}$ has presented analytical expressions for the tangent moduli related to derivatives of the fiber directions, here we use an approximation of the algorithmic tangent moduli, by considering a perturbation $0<\epsilon \ll 1$ in the deformation state which leads to

$$
A_{J i k L}=\frac{\partial P_{J i}}{\partial F_{k L}}=\lim _{\epsilon \rightarrow 0} \frac{P_{J i}\left(\boldsymbol{F}+\epsilon n_{k} \otimes N_{L}\right)-P_{i J}(\boldsymbol{F})}{\epsilon}
$$

More extensive discussions for numerical approximations of the algorithmic tangent moduli can be found in Miehe 44. 


\section{APPENDIX D: INITIAL DEFORMATION STATE}

At the initial state, the concentration of active myosin is $\tilde{c}_{a m}^{i n i t}$ which can be calculated from a steady state solution of Eq. 7 using $k_{1}=k_{0}$. The initial stretch $\lambda^{\text {init }}$ is calculated considering a stress free state of homogeneous deformation without contributions from anisotropic activation as it is zero in this unpolarized state. The deformation gradient at this state is $\boldsymbol{F}^{\text {init }}=\lambda^{\text {init }} \boldsymbol{I}$. The passive and active stress are equilibrated, where the passive stress is obtained from Eq. 26 and the active term only has a contribution from the isotropic activation defined in Eq. 29 A nonlinear equation, with $\lambda^{\text {init }}$ as the unknown, is formed adding the individual stress contributions and equating them to zero. The specific form is obtained as

$$
\frac{\mu}{2}\left(2 \lambda^{i n i t}-\frac{2}{\lambda^{i n i t}}\right)+\beta \frac{\ln \left(\left(\lambda^{i n i t}\right)^{3}\right)}{\lambda^{i n i t}}+2 \gamma^{i s o} \tilde{c}_{a m}^{i n i t}\left(\lambda^{i n i t}\right)^{5}=0
$$

and solved using a Newton-Raphson procedure.

\section{References}

[1] Kristopher Clark, Michiel Langeslag, Carl G Figdor, Frank N van Leeuwen, Trends in cell biology 2007, 17 (4), 178-186.

[2] Tadanori Mammoto, Akiko Mammoto, Donald E Ingber, Annual review of cell and developmental biology 2013, $29,27-61$.

[3] Diana E Jaalouk, Jan Lammerding, Nature reviews Molecular cell biology 2009, 10 (1), 63-73.

[4] A Wayne Orr, Brian P Helmke, Brett R Blackman, Martin A Schwartz, Developmental cell 2006, 10 (1), 11-20.

[5] Albert K Harris, David Stopak, Patricia Wild, Nature 1981, 290 (5803), 249-251.

[6] Nathan J Sniadecki, Alexandre Anguelouch, Michael T Yang, Corinne M Lamb, Zhijun Liu, Stuart B Kirschner, Yaohua Liu, Daniel H Reich, Christopher S Chen, Proceedings of the National Academy of Sciences 2007, 104 (37), 14553-14558.

[7] C Neidlinger-Wilke, ES Grood, JH-C Wang, RA Brand, L Claes, Journal of Orthopaedic Research 2001, 19 (2), $286-293$.

[8] Abhishek Tondon, Hui-Ju Hsu, Roland Kaunas, Journal of biomechanics 2012, 45 (5), 728-735.

[9] Adam J Engler, Shamik Sen, H Lee Sweeney, Dennis E Discher, Cell 2006, 126 (4), 677-689.

[10] Raimon Sunyer, Vito Conte, Jorge Escribano, Alberto Elosegui-Artola, Anna Labernadie, Léo Valon, Daniel Navajas, José Manuel García-Aznar, José J Muñoz, Pere Roca-Cusachs, et al., Science 2016, 353 (6304), 1157-1161.

[11] William J Polacheck, Christopher S Chen, Nature methods 2016, 13 (5), 415-423.

[12] Linqing Li, Jeroen Eyckmans, Christopher S Chen, Nature materials 2017, 16 (12), 1164.

[13] Christopher C DuFort, Matthew J Paszek, Valerie M Weaver, Nature reviews Molecular cell biology 2011, 12 (5), $308-319$.

[14] Julie C Friedland, Mark H Lee, David Boettiger, Science 2009, 323 (5914), 642-644.

[15] Armando del Rio, Raul Perez-Jimenez, Ruchuan Liu, Pere Roca-Cusachs, Julio M Fernandez, Michael P Sheetz, Science 2009, 323 (5914), 638-641.

[16] Magdalena Chrzanowska-Wodnicka, Keith Burridge, The Journal of cell biology 1996, 133 (6), 1403-1415.

[17] Mutsuki Amano, Masaaki Ito, Kazushi Kimura, Yuko Fukata, Kazuyasu Chihara, Takeshi Nakano, Yoshiharu Matsuura, Kozo Kaibuchi, Journal of Biological Chemistry 1996, 271 (34), 20246-20249.

[18] Benjamin Geiger, Joachim P Spatz, Alexander D Bershadsky, Nature reviews Molecular cell biology 2009, 10 (1), 21-33.

[19] Jay D Humphrey, Eric R Dufresne, Martin A Schwartz, Nature reviews Molecular cell biology 2014, 15 (12), $802-812$. 
[20] Daniel A Fletcher, R Dyche Mullins, Nature 2010, 463 (7280), 485-492.

[21] Stavros Thomopoulos, Gregory M Fomovsky, Jeffrey W Holmes, Journal of biomechanical engineering 2005, 127 (5), $742-750$.

[22] DP Nickerson, NP Smith, PJ Hunter, Philosophical Transactions of the Royal Society of London A: Mathematical, Physical and Engineering Sciences 2001, 359 (1783), 1159-1172.

[23] Vikram S Deshpande, Robert M McMeeking, Anthony G Evans, Proceedings of the National Academy of Sciences 2006, 103 (38), 14015-14020.

[24] Vikram S Deshpande, Robert M McMeeking, Anthony G Evans, in Proceedings of the Royal Society of London A: Mathematical, Physical and Engineering Sciences, The Royal Society, 2007, pp. 787-815.

[25] Franck J Vernerey, Mehdi Farsad, Journal of the mechanical behavior of biomedical materials 2011, 4 (8), $1683-1699$.

[26] William Ronan, Vikram S Deshpande, Robert M McMeeking, J Patrick McGarry, Journal of the Mechanical Behavior of Biomedical Materials 2012, 14, $143-157$.

[27] Vivek B Shenoy, Hailong Wang, Xiao Wang, Interface focus 2016, 6 (1), 20150067.

[28] A Vigliotti, W Ronan, FPT Baaijens, VS Deshpande, Biomechanics and modeling in mechanobiology 2016, 15 (4), $761-789$.

[29] Jonas Stålhand, Anders Klarbring, Gerhard A Holzapfel, Progress in biophysics and molecular biology 2008, 96 (1), $465-481$.

[30] Sae-Il Murtada, Martin Kroon, Gerhard A Holzapfel, Biomechanics and modeling in mechanobiology 2010, 9 (6), $749-762$.

[31] Jonas Stålhand, Anders Klarbring, Gerhard A Holzapfel, Journal of theoretical biology 2011, 268 (1), $120-130$.

[32] Tao Liu, in Proc. R. Soc. A, The Royal Society, 2014, p. 20130771.

[33] AV Hill, Proceedings of the Royal Society of London B: Biological Sciences 1938, 126 (843), $136-195$.

[34] Vikram S Deshpande, Milan Mrksich, Robert M McMeeking, Anthony G Evans, Journal of the Mechanics and Physics of Solids 2008, 56 (4), 1484-1510.

[35] Amit Pathak, Robert M McMeeking, Anthony G Evans, Vikram S Deshpande, Journal of Applied Mechanics 2011, 78 (4), 041001.

[36] Chi-Ming Hai, Richard A Murphy, American Journal of Physiology-Cell Physiology 1988, 254 (1), C99-C106.

[37] A Menzel, Biomechanics and modeling in mechanobiology 2005, 3 (3), 147-171.

[38] Ellen Kuhl, Krishna Garikipati, Ellen M Arruda, Karl Grosh, Journal of the Mechanics and Physics of Solids 2005, 53 (7), $1552-1573$.

[39] Paul Germain, SIAM Journal on Applied Mathematics 1973, 25 (3), 556-575.

[40] Kevin Burton, D LAnsing Taylor, Nature 1997, 385 (6615), 450-454.

[41] John L Tan, Joe Tien, Dana M Pirone, Darren S Gray, Kiran Bhadriraju, Christopher S Chen, Proceedings of the National Academy of Sciences 2003, 100 (4), 1484-1489.

[42] Mahmut Selman Sakar, Jeroen Eyckmans, Roel Pieters, Daniel Eberli, Bradley J Nelson, Christopher S Chen, Nature communications 2016, 7, 11036.

[43] Katsumi Mochitate, Pamala Pawelek, Frederick Grinnell, Experimental cell research 1991, 193 (1), $198-207$.

[44] Christian Miehe, Computer methods in applied mechanics and engineering 1996, 134 (3-4), 223-240. 